\title{
Instrumentos Padronizados para Avaliação de Aspectos Sensoriais no Bebê do Nascimento aos Seis Meses: uma ReVisáo Integrativa ${ }^{1,2}$ STANDARDIZED INSTRUMENTS FOR THE EVALUATION OF SENSORY ASPECTS IN INFANTS FROM BIRTH TO THE SIXTH MONTH: AN INTEGRATIVE REVIEW
}

\author{
Vitória Hoerbe BELTRAME ${ }^{3}$ \\ Julia Bulegon HERMES ${ }^{4}$ \\ Regina Helena Vitale Torkomian JOAQUIM ${ }^{5}$
}

\begin{abstract}
RESUMO: Este trabalho teve como objetivo identificar os instrumentos padronizados que avaliam aspectos sensoriais do nascimento aos seis meses. Trata-se de uma Revisão Integrativa de estudos publicados entre 2010 e 2020, nas bases de dados Bireme, SciElo, Scopus, Lilacs e Pubmed/Medline, além da Biblioteca Digital Brasileira de Teses e Dissertaçóes e nos periódicos Cadernos Brasileiros de Terapia Ocupacional da Universidade Federal de São Carlos, Revista de Terapia Ocupacional da Universidade de São Paulo e na Revista Interinstitucional Brasileira de Terapia Ocupacional (REVISBRATO). Adotaram-se fixamente os descritores "infant" e "sensation disorders", o termo livre "scale" e o operador boleano "AND" e, no formato alternado, os termos livres "Sensory Profile", "Sensory Functions" e "Sensory Processing". Incluíram-se estudos em português, inglês e espanhol. Estudos de revisão, pesquisas com profissionais e que não correspondiam à temática foram excluídos dessa revisão. Foram selecionados seis estudos. Entre os oito instrumentos identificados, apenas o Infant/Toddler Sensory Profile e o Test of Sensory Functions Infants avaliam especificamente aspectos sensoriais. Identificou-se o uso de dois instrumentos de avaliaçáo do contexto em que a criança está inserida para avaliar aspectos sensoriais como o HOME e o AHEMED e outros instrumentos de análise geral do desenvolvimento como a Escala Bayley III. Há necessidade de ampliação do uso de instrumentos específicos de análise do processamento sensorial para aumentar as oportunidades de familiares, professores e profissionais a apoiar o desenvolvimento do bebê, além de identificar sinais de risco que, quando tratados, diminuem os efeitos iatrogênicos no desenvolvimento.
\end{abstract}

PALAVRAS-CHAVE: Instrumentos de avaliação. Experiência sensorial. Primeira infância. Revisão de literatura.

\begin{abstract}
This work aimed to identify the standardized instruments that assess sensory aspects in infants from birth to the sixth month. It is an Integrative Review of studies published from 2010 to 2020 in Bireme, SciElo, Scopus, Lilacs and Pubmed/Medline databases, in the Brazilian Digital Library of Theses and Dissertations, and in the Brazilian Journal of Occupational Therapy from the Universidade Federal de São Carlos, in the Journal of Occupational Therapy from the University of São Paulo and the Interinstitutional Brazilian Journal of Occupational Therapy (REVISBRATO). The descriptors "infant" and "sensation disorders", the free term "scale", and the Boolean operator "AND" were fixedly adopted, whereas the free terms "Sensory Profile", "Sensory Functions" and "Sensory Processing" were alternately adopted. Studies in Portuguese, English and Spanish were included. Review studies, researches with professional and others that did not correspond to the theme were excluded from this review. Six studies were selected. Among the eight identified instruments, only the Infant/Toddler Sensory Profile and the Test of Sensory Functions in Infants specifically assess sensory aspects. The use of two instruments for the assessment of the context in which the infant is inserted to assess sensory aspect, as HOME and AHEMED, were identified, as well as other instruments for general analysis of development, as Bayley-III. There is the need to broaden the use of specific instruments for the analysis of sensory processing in order to increase the opportunities for family members, teachers and professionals to support the infant development, as well as to identify risk signs that, when treated, decrease iatrogenic effects in development.
\end{abstract}

KEYWORDS: Assessment instruments. Sensory experience. Early childhood. Literature review.

\footnotetext{
${ }^{1}$ https://doi.org/10.1590/1980-54702021v27e0149

${ }^{2}$ Trabalho realizado com apoio da Coordenação de Aperfeiçoamento de Pessoal de Nível Superior - Brasil (CAPES) - Código de Financiamento 001

${ }^{3}$ Departamento de Terapia Ocupacional da Universidade Federal de Santa Maria (UFSM). Santa Maria/Rio Grande do Sul/Brasil. E-mail: vitoria.beltrame@ufsm.br. ORCID: https://orcid.org/0000-0002-1156-9572

${ }^{4}$ Departamento de Terapia Ocupacional da Universidade Federal de Santa Maria (UFSM). Santa Maria/Rio Grande do Sul/Brasil. E-mail: juliabhermes@gmail.com. ORCID: https://orcid.org/0000-0002-3854-8093

${ }^{5}$ Departamento de Terapia Ocupacional da Universidade Federal de São Carlos (UFSCar). São Carlos/São Paulo/Brasil. E-mail: joaquimrhvt@gmail.com. ORCID: https://orcid.org/0000-0003-3700-397X
} 


\section{INTRODUÇÁo}

O processo de maturação neurológica inicia-se no espaço intrauterino com o refinamento dos sistemas sensoriais que gera qualidade ao crescimento cerebral e repercute na vida do bebê após o nascimento (Gonçalves \& Goto, 2010). Nos primeiros anos de vida, as aquisiçóes do desenvolvimento evoluem rapidamente de forma que se caracterizam com reaçôes provenientes do sistema vestibular, gustativo, visual, auditivo, tátil e proprioceptivo (Almohalha, 2018). Os sistemas sensoriais quando bem integrados possibilitam um adequado processamento sensorial, o que somado aos avanços do desenvolvimento infantil como um todo, por meio de aspectos motores, cognitivos, psíquicos e sociais, ampliam as capacidades do bebê para interagir com o meio e adquirir habilidades em todas as áreas de desempenho. A possibilidade de integrar as sensaçōes corporais com as informaçōes advindas do meio externo e do Sistema Nervoso Central capacita o sujeito para responder de forma adequada aos estímulos recebidos, caracterizando um adequado processamento sensorial (Almohalha, 2018).

Atualmente, os aspectos sensoriais devem ser considerados tanto quanto outras áreas nas avaliaçóes de desenvolvimento infantil, e isso se torna ainda mais importante quando tratamos de avaliação com bebês, pois, após o nascimento, inicia-se o período de maior neuroplasticidade: a fase em que o sistema nervoso tem a maior capacidade de se organizar e reestruturar funcionalmente em decorrência de adaptaçóes exigidas pelo meio (Gioia \& Guilhardi, 2018). Portanto, quanto mais cedo são identificados os fatores de risco e o modo como isso influencia o desenvolvimento do bebê, aumenta-se as chances de intervenção, contribuindo com a diminuiçáo de comprometimentos (Gioia \& Guilhardi, 2018) e a melhora na participação e no desempenho nas atividades diárias e ocupaçóes (Almohalha, 2018).

A ampliação no campo de conhecimento de sinais de risco no desenvolvimento de forma muito precoce em relação a aspectos sensoriais é observado em estudos que se propuseram a analisar grupos de bebês nascidos pré-termo, apresentando evidências científicas que esse grupo possui maior tendência a apresentar Transtornos do Processamento Sensorial (Machado et al., 2017; Morimoto et al., 2020).

Além da população de crianças nascidas prematuramente, outros grupos de risco para atraso no desenvolvimento de uma forma geral, sejam por questóes neurológicas, síndromes ou risco psíquico, já estão sendo referenciadas na literatura como pré-disponíveis a risco no que tange também aos aspectos sensoriais (Beltrame et al., 2018; Bröring et al., 2018; Buffone et al., 2016; Goméz et al., 2019; Reis et al., 2018). Os Transtornos de Processamento Sensorial podem, durante a infância, interferir no desempenho em atividades do dia a dia como as tarefas de aprendizagem no contexto escolar, na funcionalidade, na atenção e na concentraçáo, bem como no desempenho, evidenciado em estudos que se propuseram a estudar a Integração Sensorial de crianças com dificuldades de aprendizagem nos contextos escolares, Transtorno do Espectro Autista (TEA), diagnósticos neurológicos e Síndromes.

Essas dificuldades muitas vezes tornam-se evidentes com a inserção da criança na escola e devem ser identificadas precocemente (Bröring et al., 2018), já que, após percebidas, exigem o planejamento de estratégias e intervençóes conjuntas entre terapeutas ocupacionais, professores(as) e educadores(as) especiais (Pisacco et al., 2016). O planejamento conjunto é fundamental para identificar as potencialidades da criança e os apoios necessários para que 
realize qualquer experiência cotidiana em casa e na escola. Isso, por vezes, repercute em adaptaçóes ambientais, modificação nos currículos escolares, utilização de recursos de apoio, adequação entre o tempo de oferta e respostas a tarefas, modificaçóes nas instruçóes para realização de uma atividade e flexibilização de rotinas (Pisacco et al., 2016).

Esse panorama tem influenciado cada vez mais os profissionais da área da infância a produzir conhecimento científico sobre o desenvolvimento infantil que considere o bebê também sob uma perspectiva sensorial (Aragão et al., 2018; Buffone et al., 2016; Guadarrama-Celaya, 2012; Morimoto et al., 2020; Souza et al., 2019), porém ainda há uma defasagem de instrumentos padronizados específicos que possam vir a detalhar comportamentos e/ou sinais de risco sobre as possíveis relaçóes entre o processamento sensorial e os comportamentos no bebê.

Instrumentos de medidas padronizados são aqueles com características de avaliação uniformes que justificam a confiabilidade dos dados que produzem (Echevarría-Guanilo et al., 2017). O instrumento deve ser bem formulado com normas claras de aplicação e com resultados/escores quantificados de forma que a adaptação para idiomas e culturas diferentes é fundamental e deve manter suas propriedades quanto à validade e à confiabilidade após a adaptação (Echevarría-Guanilo et al., 2017).

Atualmente, os Terapeutas Ocupacionais brasileiros estão se dedicando a qualificar os instrumentos avaliativos de áreas sensoriais para a população infantil, tendo visto que, no ano de 2018, foi realizada a tradução, a adaptação cultural e a validação do Instrumento Infant/ Toddler Sensory Profile 2 (Dunn \& Daniels, 2002) para crianças brasileiras de 0 a 35 meses, nomeado no Brasil como Perfil Sensorial 2 (Almohalha, 2018). O instrumento é fundamentado em questóes cotidianas que objetivam compreender a percepção dos pais sobre as experiências sensoriais do bebê ao longo do dia, produzindo informaçóes importantes aos avaliadores, já que os primeiros conceitos relativos às sensaçóes vividas pelo bebê sobre si e sobre o mundo são oriundos de seus cuidadores (Peruzzolo \& Souza, 2017) de forma que as respostas a essas estimulações, advindas do cotidiano, devem servir como embasamento para avaliaçóes principalmente no que tange ao processamento sensorial. Os escores finais do instrumento Perfil Sensorial 2 apontam resultados indicativos sobre o processamento sensorial do bebê e seus efeitos no desempenho funcional das atividades diárias da criança, indicando a necessidade de acompanhamento ou não em serviços de follow-up (Almohalha, 2018).

Ainda assim, no Brasil, há escassez de dados normativos e instrumentos padronizados e validados para avaliar aspectos sensoriais na infância, principalmente na faixa etária que compreende a primeira infância (Almohalha, 2018), mais especificamente do nascimento aos seis meses. A revisão sistemática publicada por Machado et al. (2017) que apresentou resultados de artigos publicados entre os anos de 2005 e 2015, apontou evidências científicas nesse período quanto ao processamento sensorial em crianças nascidas pré-termo no período da infância, resultando em oito artigos, dos quais apenas dois utilizaram instrumentos padronizados para avaliação específica do processamento sensorial em bebês do nascimento aos seis meses.

Já em estudos de revisão que se propuseram a apresentar instrumentos de avaliação de desenvolvimento para bebês (Mélo et al., 2019; Silva et al., 2011), percebe-se a caracterização de uma ampla quantidade de instrumentos padronizados para avaliação do desenvolvimento infantil como a Escala Bayley de desenvolvimento de bebês e crianças - Bayley III, Teste de 
Denver II, Alberta Motor Infant Scale (AIMS). Contudo, nesses mesmos estudos, nenhum instrumento caracterizado tem como objetivo obter resultados quanto ao processamento sensorial de uma forma específica e prioritária, já que o foco dos instrumentos citados estão relacionados principalmente a avaliar aspectos cognitivos, motores, psicomotores e de linguagem (Mélo et al., 2019; Silva et al., 2011).

Diante do exposto, o objetivo deste estudo foi identificar quais são os instrumentos padronizados utilizados para avaliação de aspectos sensoriais em bebês do nascimento aos seis meses de idade. Pretendeu-se também caracterizar os instrumentos padronizados quanto aos aspectos sensoriais avaliados, a aplicação por faixas etárias, os escores estabelecidos e a existência de validação e adaptação para população brasileira. Além disso, descrever os estudos que fizeram uso desses instrumentos quanto ao título, ao ano de publicação, ao objetivo, à população, à metodologia e aos resultados, e, também, identificar se outros instrumentos de avaliação do desenvolvimento cognitivo, motor e psíquico estão sendo utilizados para identificar aspectos sensoriais e de que forma isso está sendo apresentado na literatura científica.

\section{Metodologia}

Este estudo caracteriza-se como uma Revisão Integrativa. Os estudos do tipo Revisão Integrativa baseiam-se em um método utilizado na Prática Baseada em Evidências (PBE) e são fundamentados na busca de publicaçóes recentes, com o objetivo de consolidar o conhecimento e o apontamento de lacunas em relação ao tema estudado (Souza et al., 2010). Esse tipo de revisão permite a junção entre estudos experimentais e não-experimentais, de modo que o objetivo final é a compreensão do tema estudado reunindo o maior número de informaçóes possíveis (Souza et al., 2010). As etapas para construção deste estudo foram: 1 - Elaboração da pergunta norteadora; 2 - Busca ou amostragem na literatura; 3 - Coleta de dados; 4 Análise crítica dos artigos incluídos; 5 - Discussão dos resultados; 6 - Apresentação da Revisão Integrativa (Souza et al., 2010).

A partir dos passos apontados, a pergunta de pesquisa foi: "Quais instrumentos padronizados estão sendo usados para avaliação de aspectos sensoriais em bebês de 0 a 6 meses?”. A partir disso, iniciou-se o levantamento dos artigos por meio de duas pesquisadoras independentes nas bases de dados Bireme, SciElo, Scopus, Lilacs e Pubmed/Medline. Realizou-se também o levantamento de Teses e Dissertaçôes referentes à temática na Biblioteca Digital Brasileira de Teses e Dissertaçôes (BDTD). Para os cruzamentos, foi utilizado o operador boleano "AND” por meio dos Descritores em Saúde (DECs) "infant" e "sensation disorders", além dos termos livre "Scale", "Sensory Profile", "Sensory Functions" e "Sensory Processing". Os descritores e os termos livres foram cruzados em inglês, português e espanhol em todas as bases de dados, como exposto na Figura 1. Além da busca nas bases de dados, foram realizados levantamentos a partir dos títulos de artigos nos Cadernos Brasileiros de Terapia Ocupacional da Universidade Federal de São Carlos (UFSCar), Revista de Terapia Ocupacional da Universidade de São Paulo (USP) e na Revista Interinstitucional Brasileira de Terapia Ocupacional (REVISBRATO).

Após os cruzamentos realizados nas bases de dados, as pesquisadoras compararam os estudos encontrados, definindo de forma conjunta quais se manteriam para análise na íntegra. O mesmo foi feito nas buscas realizadas nas Revistas brasileiras de Terapia Ocupacional, de 
modo que, após a análise dos títulos e dos resumos dos estudos para identificação dos critérios de inclusão e de exclusão, compararam-se os resultados entre as pesquisadoras. Quando houve discordância em relação à inclusão ou à exclusão de algum estudo, estes foram lidos na íntegra por ambas as pesquisadoras alcançando, assim, um posicionamento comum.

Os critérios de inclusão foram: estudos que incluíram na amostra bebês de 0 a 6 meses, o uso de instrumento padronizado e artigos publicados entre 2010 e 2020. Os critérios que excluíram os estudos encontrados foram: estudos que não abordaram a temática desta pesquisa, estudos de revisão e estudos com profissionais.

As buscas por meio dos cruzamentos nas bases de dados localizaram 146 estudos e o processo realizado nas bases de dados está exposto na Figura 1. A busca livre nas Revistas Brasileiras de Terapia Ocupacional identificou 18 estudos possíveis de serem incluídos, totalizando 164 estudos. Com a leitura e a identificação dos critérios de inclusão e exclusão, mantiveram-se, na presente revisão para análise, seis títulos, sendo eles uma dissertaçáo de Mestrado e cinco artigos.

Para a análise dos artigos incluídos, foi utilizado, inicialmente, o instrumento de coleta de dados validado por Ursi e Galvão (2006) e, posteriormente, realizou-se uma síntese dos artigos que atenderam aos critérios de inclusão que serão apresentados nos resultados por meio de um quadro. Essa síntese contemplou os seguintes aspectos: identificação dos autores, ano de publicação, título e objetivo, metodologia, instrumentos padronizados e resultados.

A apresentação dos resultados e a discussão dos dados obtidos foi feita de forma descritiva, possibilitando ao leitor a avaliação da aplicabilidade da revisão integrativa elaborada, de forma a atingir o objetivo desse método: impactar positivamente na qualidade da prática de profissionais das áreas de saúde e educação que atuam com bebês. 
BELTRAME, V.H.; HERMES, J.B.; JOAQUIM, R.H.V.T.

\section{Figura 1}

Fluxograma de seleção dos artigos incluidos na revisão
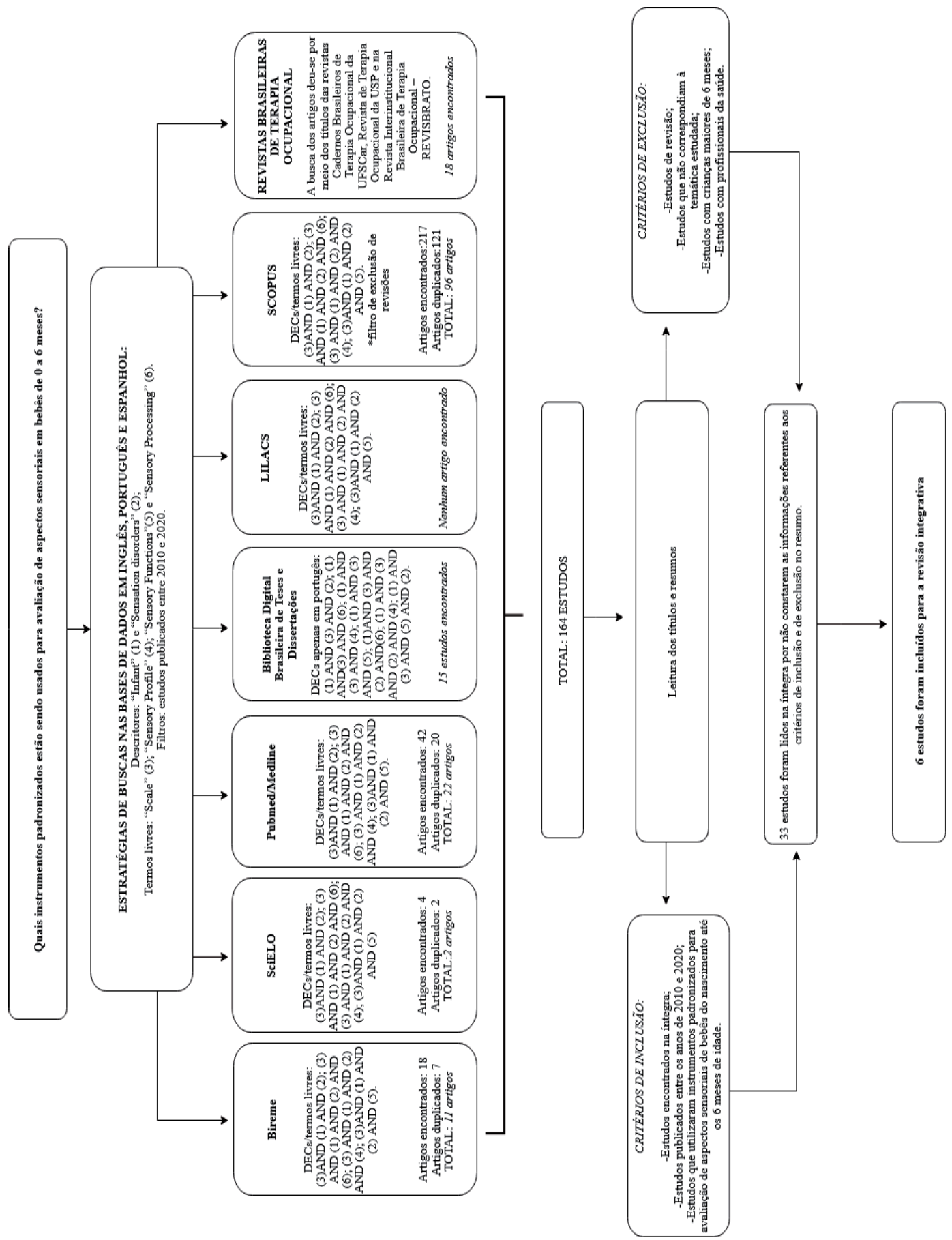


\section{Resultados/discussão}

$\mathrm{Na}$ presente revisão integrativa, foram analisados seis estudos que atenderam aos critérios de inclusão previamente estabelecidos e, a seguir, no Quadro 1, apresentar-se-á um panorama geral dos estudos identificados.

\section{Quadro 1}

\section{Caracterização dos artigos incluidos na revisäo integrativa}

\begin{tabular}{|c|c|c|c|c|c|c|}
\hline Autores(as) & Título & Ano & Objetivos & $\begin{array}{l}\text { Metodologia/ } \\
\text { Tipo de estudo }\end{array}$ & Instrumentos & Principais resultados \\
\hline $\begin{array}{l}\text { Campos, A. C., } \\
\text { Coelho, M. C., } \\
\text { \& Rocha, N. A. } \\
\text { C. F. }\end{array}$ & $\begin{array}{l}\text { Desempenho } \\
\text { motor e senso- } \\
\text { rial de lactentes } \\
\text { com e sem } \\
\text { síndrome de } \\
\text { Down: estudo } \\
\text { piloto }\end{array}$ & 2010 & $\begin{array}{l}\text { Avaliar o perfil } \\
\text { motor e sensorial } \\
\text { de lactentes com } \\
\text { Síndrome de Down } \\
\text { em comparaçáo com } \\
\text { lactentes típicos aos } \\
6 \text { meses de idade }\end{array}$ & $\begin{array}{l}\text {-Estudo piloto } \\
\text { com caráter } \\
\text { transversal; } \\
\text {-Amostra: } 8 \\
\text { lactentes, } 4 \text { bebês } \\
\text { com Síndrome } \\
\text { de Down (SD) e } \\
4 \text { lactentes com } \\
\text { desenvolvimento } \\
\text { típico aos } 6 \\
\text { meses }\end{array}$ & $\begin{array}{l}\text {-Infant/Toddler } \\
\text { Sensory Profile } \\
\text { (ITSP) - Avaliação } \\
\text { do Desempenho } \\
\text { sensorial; } \\
\text {-Alberta Infant } \\
\text { Motor Scale (AIMS) } \\
\text { - Escala Motora } \\
\text { Infantil de Alberta }\end{array}$ & $\begin{array}{l}\text { Os resultados no ITSP mostram que os lacten- } \\
\text { tes com SD obtiveram piores escores em baixo } \\
\text { registro, possivelmente por apresentarem altos } \\
\text { limiares neurológicos, } \\
\text { demorando mais para responder aos estímu- } \\
\text { los. Na AIMS, os lactentes com SD tiveram } \\
\text { um desempenho inferior quando comparados } \\
\text { aos típicos na subescala prono, o que pode } \\
\text { advir de dificuldades no controle postural e } \\
\text { antigravitacional. Năo foi } \\
\text { encontrada correlação entre os escores do } \\
\text { ITSP e da AIMS. }\end{array}$ \\
\hline $\begin{array}{l}\text { Avan, B. I., } \\
\text { Raza, S. A., \& } \\
\text { Kirkwood, B. R. }\end{array}$ & $\begin{array}{l}\text { A communi- } \\
\text { ty-based study } \\
\text { of early child- } \\
\text { hood sensory } \\
\text { stimulation in } \\
\text { home environ- } \\
\text { ment associated } \\
\text { with growth and } \\
\text { psychomotor } \\
\text { development in } \\
\text { Pakistan }\end{array}$ & 2014 & $\begin{array}{l}\text { Avaliar a estimu- } \\
\text { lação sensorial no } \\
\text { ambiente doméstico } \\
\text { e examinar a sua } \\
\text { influência no } \\
\text { crescimento físico e } \\
\text { no desenvolvimento } \\
\text { psicomotor }\end{array}$ & $\begin{array}{l}\text {-Estudo } \\
\text { transversal em } \\
26 \text { comunidades } \\
\text { da província } \\
\text { de Sindh no } \\
\text { Paquistấo. } \\
\text {-Amostra: } 1.244 \\
\text { crianças; } 454 \\
\text { apresentavam } \\
\text { entre } 0 \text { e um } \\
\text { ano, } 454 \text { apre- } \\
\text { sentavam entre } \\
\text { um e dois anos e } \\
336 \text { estavam na } \\
\text { faixa etária entre } \\
\text { dois e três anos } \\
\text { de idade }\end{array}$ & $\begin{array}{l}\text {-Escala Bayley de } \\
\text { Desenvolvimento } \\
\text { Infantil III } \\
\text {-Home Observation } \\
\text { for Measurement } \\
\text { of the Environment } \\
\text { (HOME) }\end{array}$ & $\begin{array}{l}\text { Nas zonas rurais, a estimulaçáo sensorial } \\
\text { fornecida foi menor quando comparada às ur- } \\
\text { banas e mostrou associaçáo com baixa estatura } \\
\text { e baixo peso, não explicada pelo tipo de bairro } \\
\text { ou status socioeconômico. A estimulaçáo } \\
\text { sensorial esteve associada ao desenvolvimento } \\
\text { psicomotor mais do que à contribuiçáo } \\
\text { combinada do status socioeconômico e dos } \\
\text { fatores rural-urbanos. }\end{array}$ \\
\hline $\begin{array}{l}\text { Chorna, O., } \\
\text { Solomon, J. E., } \\
\text { Slaughter, J. C., } \\
\text { Stark, A. R., \& } \\
\text { Maitre, N. L. }\end{array}$ & $\begin{array}{l}\text { Abnormal } \\
\text { sensory reactiv- } \\
\text { ity in preterm } \\
\text { infants during } \\
\text { the first year } \\
\text { correlates with } \\
\text { adverse neuro- } \\
\text { developmental } \\
\text { outcomes at } 2 \\
\text { years of age }\end{array}$ & 2014 & $\begin{array}{l}\text { Avaliar o Processa- } \\
\text { mento Sensorial e } \\
\text { sua associaçáo com } \\
\text { o neurodesenvolvi- } \\
\text { mento }\end{array}$ & $\begin{array}{l}\text {-Estudo } \\
\text { observacional, } \\
\text { prospectivo; } \\
\text { - Amostra: } 72 \\
\text { bebês com idade } \\
\text { corrigida entre } 4 \\
\text { a } 12 \text { meses. }\end{array}$ & $\begin{array}{l}\text {-Test of Sensory } \\
\text { Function in Infants } \\
\text { (TSFI); } \\
\text {-Developmental } \\
\text { Assessment of Young } \\
\text { Children (DAYC); } \\
\text {-Escala Bayley de } \\
\text { Desenvolvimento } \\
\text { Infantil III (EBDI } \\
\text { III) }\end{array}$ & $\begin{array}{l}59 \text { bebês }(82 \%) \text { apresentaram pelo menos um } \\
\text { dos escores no TSFI referente à reatividade } \\
\text { sensorial anormal. A menor idade gestacional } \\
\text { foi associada à reatividade anormal quanto a } \\
\text { pressão profunda e à estimulaçáo vestibular. O } \\
\text { controle oculomotor inadequado previa piores } \\
\text { escores cognitivos e motores na primeira } \\
\text { infância, mas estave fortemente correlacionado } \\
\text { à presença de lesão grave na substância branca. } \\
\text { Os escores baixos apresentados pelos bebês na } \\
\text { funçáo motora adaptativa esteve associado a } \\
\text { pior desempenho motor e de linguagem na } \\
\text { Escala Bayley de Desenvolvimento Infantil } \\
\text { aos dois anos. }\end{array}$ \\
\hline $\begin{array}{l}\text { Pedrosa, C., } \\
\text { Caçola, P., \& } \\
\text { Carvalhal, M. } \\
\text { I. M. }\end{array}$ & $\begin{array}{l}\text { Fatores } \\
\text { preditores do } \\
\text { perfil sensorial } \\
\text { de lactentes dos } \\
4 \text { aos } 18 \text { meses } \\
\text { de idade }\end{array}$ & 2015 & $\begin{array}{l}\text { Identificar os fatores } \\
\text { ambientais } \\
\text { preditores do } \\
\text { perfil sensorial dos } \\
\text { lactentes com idades } \\
\text { entre } \\
\text { quatro e } 18 \text { meses e } \\
\text { caracterizar o con- } \\
\text { texto familiar e de } \\
\text { creche quanto às } \\
\text { affordances }\end{array}$ & $\begin{array}{l}\text { Estudo trans- } \\
\text { versal; } \\
3 \text { amostras por } \\
\text { conveniência; } \\
\text { Amostra 1: } 97 \\
\text { bebês; Amostra } \\
\text { 2: } 97 \text { pais; } \\
\text { Amostra 3: } 11 \\
\text { educadores de } \\
\text { sete creches. }\end{array}$ & $\begin{array}{l}\text {-Test of Sensory } \\
\text { Functions in Infants } \\
\text { (TSFI) } \\
\text {-Affordance in the } \\
\text { Home Environment } \\
\text { for Motor Develop- } \\
\text { ment- Infant Scale } \\
\text { (AHEMD-IS) }\end{array}$ & $\begin{array}{l}\text { A maioria dos bebês ( } 66 \%) \text { apresentou } \\
\text { um perfil sensorial normal e } 34 \% \text { deles } \\
\text { encontram-se em risco ou em déficit. As opor- } \\
\text { tunidades de estimulaçáo na habitaçáo foram } \\
\text { classificadas como suficientes e nas creches } \\
\text { foram avaliadas como boas. Os resultados } \\
\text { da regressáo revelaram que apenas os fatores } \\
\text { "horas diárias na creche" e "espaço exterior de } \\
\text { creche" influenciaram o perfil sensorial dos } \\
\text { bebês, principalmente } \\
\text { o controle oculomotor. }\end{array}$ \\
\hline
\end{tabular}




\begin{tabular}{|c|c|c|c|c|c|c|}
\hline Autores(as) & Título & Ano & Objetivos & $\begin{array}{l}\text { Metodologia/ } \\
\text { Tipo de estudo }\end{array}$ & Instrumentos & Principais resultados \\
\hline $\begin{array}{l}\text { Cavaggioni, A. } \\
\text { P. M. }\end{array}$ & $\begin{array}{l}\text { Influência da } \\
\text { via de parto no } \\
\text { desenvolvi- } \\
\text { mento infantil: } \\
\text { comparaçáo por } \\
\text { meio da escala } \\
\text { Bayley III }\end{array}$ & 2017 & $\begin{array}{l}\text { Avaliar o desenvol- } \\
\text { vimento psicoló- } \\
\text { gico e comparar o } \\
\text { desenvolvimento } \\
\text { cognitivo, de } \\
\text { linguagem, motor, } \\
\text { socioemocional e } \\
\text { comportamentos } \\
\text { adaptativos de bebês } \\
\text { de } 0 \text { a } 42 \text { meses, } \\
\text { nascidos de cesárea } \\
\text { eletiva e parto } \\
\text { normal }\end{array}$ & $\begin{array}{l}\text { Estudo quanti- } \\
\text { tativo; Amostra } \\
\text { por conveniên- } \\
\text { cia: } 263 \text { crianças } \\
\text { com idades entre } \\
6 \text { e } 42 \text { meses } \\
\text { foram avaliadas } \\
\text { em creches do } \\
\text { município de } \\
\text { São Bernardo } \\
\text { dos Campos }\end{array}$ & $\begin{array}{l}\text {-Escala Bayley de } \\
\text { Desenvolvimento } \\
\text { Infantil III }\end{array}$ & $\begin{array}{l}\text { Os resultados apontaram a via de parto e } \\
\text { a idade gestacional ao nascer como fatores } \\
\text { de risco ao desenvolvimento psicológico da } \\
\text { criança. Entre os bebês nascidos por parto } \\
\text { cesáreo, } 12 \% \text { apresentaram desempenho infe- } \\
\text { rior em relaçáo ao processamento sensorial, e } \\
10 \text { a } 19 \% \text { nas habilidades do comportamento } \\
\text { adaptativo. Nas correlaçóes com a idade gesta- } \\
\text { cional ao nascer, também se observou diferen- } \\
\text { ça estatisticamente significativa (p<0,05): } 12 \% \\
\text { dos nascidos a termo entre } 37 \text { e } 38 \text { semanas } \\
\text { de idade gestacional apresentaram resultados } \\
\text { inferiores no desenvolvimento da linguagem } \\
\text { expressiva e } 9 \% \text { na motricidade fina. }\end{array}$ \\
\hline $\begin{array}{l}\text { Guadarrama-Ce- } \\
\text { laya, F.. Otero- } \\
\text {-Ojeda, G. A., } \\
\text { Pliego-Rivero, } \\
\text { F. B., Porcayo- } \\
\text {-Mercado, M. } \\
\text { R., Ricardo- } \\
\text {-Garcell, J., \& } \\
\text { Pérez-Ábalo, } \\
\text { M. C. }\end{array}$ & $\begin{array}{l}\text { Screening of } \\
\text { Neurodevelop- } \\
\text { mental } \\
\text { Delays in Four } \\
\text { Communities } \\
\text { of Mexico and } \\
\text { Cuba }\end{array}$ & 2012 & $\begin{array}{l}\text { Avaliar se a preva- } \\
\text { lência de desvios } \\
\text { de neurodesenvol- } \\
\text { vimento normal } \\
\text { diferiu entre as } \\
\text { comunidades } \\
\text { mexicanas e cubanas } \\
\text { e a viabilidade da } \\
\text { introduçáo da ferra- } \\
\text { menta NPED em } \\
\text { centros de saúde } \\
\text { locais }\end{array}$ & $\begin{array}{l}\text {-Estudo } \\
\text { observacional e } \\
\text { transversal; } \\
\text {-Amostra: } 400 \\
\text { crianças de } 1 \text { a } \\
60 \text { meses de } 4 \\
\text { comunidades do } \\
\text { México e Cuba }\end{array}$ & $\begin{array}{l}\text { Neuropediatric } \\
\text { Development } \\
\text { (NPED) }\end{array}$ & $\begin{array}{l}\text { Foram observadas falhas em linguagem, } \\
\text { aspectos psicomotores e sensoriais visuais } \\
\text { e auditivas para a toda a amostra. Entre as } \\
\text { comunidades mexicanas houveram falhas } \\
\text { apenas no teste auditivo da populaçáo da } \\
\text { comunidade urbana. Quando comparada, a } \\
\text { comunidade cubana mostrou uma proporção } \\
\text { significativamente maior de falhas na audiçâo } \\
\text { em relação às comunidades mexicanas. }\end{array}$ \\
\hline
\end{tabular}

Os artigos incluídos nesta revisão utilizaram oito instrumentos diferentes para coleta de dados, como exposto no Quadro 1. Entretanto, apenas dois têm como proposta a avaliação de aspectos sensoriais, a saber: Infant/Toddler Sensory Profile (ITSP)/Perfil Sensorial do bebê e da criança e Test of Sensory Functions Infants (TSFI). Considerando o objetivo deste estudo, será apresentada uma breve descrição dos dois instrumentos e, para isso, foram definidas duas categorias: Aspectos sensoriais - Avaliação do bebê; e Aspectos sensoriais do bebê - Questionário com os cuidadores. Uma terceira categoria foi criada para apresentar o uso de instrumentos de avaliação das áreas motora, cognitiva e psíquica que foram utilizadas nos estudos, com o objetivo de identificar aspectos sensoriais em bebês de 0 a 6 meses.

\subsection{AsPeCtos SENSORIAIS - AVALIAÇÃo Do BEBÊ}

O Test of Sensory Functions in Infants (TSFI) (DeGangi \& Greenspan, 1989) foi desenvolvido para aplicação com bebês entre 4 e 18 meses. O instrumento é composto por 24 itens que são avaliados individualmente e fornecem uma medida geral do processamento e da reatividade sensorial para lactentes. $\mathrm{O}$ teste propóe-se a avaliar cinco subdomínios, sendo eles: Reatividade à pressão profunda - Análise do processamento tátil; Funçóes motoras adaptativas - Identificaçáo das capacidades de planejamento motor e a forma como interage com os objetos oferecidos; Integração da visão com o tato - Avaliação das capacidades táteis associadas às capacidade visuais em atividades funcionais; Controle oculomotor - capacidades visuais na exploração; Reação a estímulos vestibulares nos planos vertical e horizontal, bem como a presença ou não de nistagmo (Pedrosa et al., 2015). Conforme as informaçóes originadas, os escores totais são produzidos considerando três tipos de resposta: normal, em risco e deficiente, que se referem tanto à classificação individual dos subdomínios, como ao resultado da pontuação total.

É possível perceber no Quadro 1 que dois artigos incluídos utilizaram o TSFI como instrumento de coleta de dados, dos quais um se propôs a comparar o processamento sensorial 
entre bebês típicos, e outro realizou a comparação entre bebês nascidos prematuros e a termo. Buffone et al. (2016) e Chorna et al. (2014) compararam o processamento sensorial de bebês e identificaram que crianças nascidas prematuras apresentaram mais dificuldades no processamento sensorial quando comparadas aos bebês a termo. Diferentemente do estudo de Pedrosa et al. (2015), em que as autoras avaliaram crianças típicas dos quatro aos 18 meses por meio do TSFI e identificaram que o instrumento foi sensível na identificação de sinais de risco no processamento sensorial em $34 \%$ da população estudada, classificando-a em risco ou em déficit. Neste estudo, as crianças apresentaram piores escores nas subescalas de funções motoras adaptativas e em relação às reações à estimulação vestibular.

Uma revisão sistemática realizada por Terapeutas Ocupacionais com o objetivo de identificar evidências sobre o processamento sensorial de crianças nascidas prematuramente identificou três estudos publicados com dados referentes ao TSFI (Machado et al., 2017), instrumento que, para muitos autores, é considerado como de excelência quanto à validade e à confiabilidade (Machado et al., 2017). Ressalta-se que um dos estudos que utilizou o TSFI é brasileiro, o que pode justificar as diferenças entre os resultados observados nas pesquisas com bebês nascidos prematuros, já que náo há validação e adaptaçáo do instrumento para a população brasileira.

\subsection{AspeCtos DENSORIAIS DO BEBÊ - QUESTIONÁRIO COM OS CUIDADORES}

O Infant/Toddler Sensory Profile - ITSP (Duun \& Daniels, 2002) foi um dos instrumentos utilizados em um dos estudos para avaliar o perfil sensorial de lactentes com Síndrome de Down em comparação com lactentes típicos, como apontado no Quadro 1. Foi desenvolvido para aplicaçáo do nascimento aos 35 meses do bebê no formato de um questionário com pais ou cuidadores. Para essa faixa etária, o instrumento ainda tem diferenciaçóes, visto que dos 0 a seis meses é nomeado de Infant Sensory Profile. Apresenta como objetivo traçar o perfil do processamento sensorial do bebê a partir da percepção dos pais, que, por meio de uma escala Likert, respondem sobre a frequência com que cada comportamento é percebido no cotidiano. As seis categorias avaliadas pelo instrumento são: processamento geral, processamento auditivo, processamento visual, processamento tátil, processamento vestibular e processamento sensorial oral.

Os resultados do estudo de Campos et al. (2010) relataram que o uso do instrumento Infant Sensory Profile indicou piores escores dos lactentes com Síndrome de Down em comparação aos bebês típicos na subescala de baixo registro para estímulos, porém não foram identificadas diferenças entre os grupos quanto à excessiva sensibilidade a estímulos bem como a comportamentos, como buscar ou evitar estímulos sensoriais.

O estudo de Campos et al. (2010), caracterizado no Quadro 1, foi publicado no ano de 2010. Com a tradução e a validação do instrumento para a populaçáo brasileira no ano de 2018 (Almohalha, 2018), faz-se necessária a realização de estudos que se proponham a utilizá-lo na prática clínica e em pesquisas, apresentando escores condizentes com as características dessa população, já que os resultados apresentados por Almohalha (2018) demonstram as diferenças na tradução dos itens do instrumento como também nos escores finais. As modificaçóes apresenta- 
das na tradução de um instrumento e nos escores finais, consideradas a partir das características singulares de uma determinada população, podem implicar significativamente os resultados de um estudo, o que deve ser levado em consideração ao analisar-se o artigo de Campos et al. (2010) que apontam essa questáo como fator limitante nos resultados do seu estudo.

Desde 2018, tendo o instrumento traduzido e validado para a população brasileira, ressalta-se a importância de que os Terapeutas Ocupacionais possam utilizá-lo na prática clínica, visto que os cuidadores são informantes fundamentais do processo de desenvolvimento do bebê (Beltrame et al., 2018; Joaquim et al., 2018). Devem, também, ser considerados de forma singular nas avaliaçóes, assim como defenderam Gioia e Guilhardi (2018) quando propuseram a elaboração de um protocolo de identificação precoce de TEA e buscaram informações com os pais sobre os objetos de interesses do bebê, as maneiras de comunicação do bebê com os pais e o repertório comportamental do bebê diante de diferentes situaçóes cotidianas. Della Barba (2018) defende que a valorização do protagonismo da família nas açôes que envolvem a criança alvo do processo deve ser considerada e efetivada, afirmando, portanto, a importância de que as avaliaçóes do desenvolvimento infantil devam ser fundamentadas considerando as observaçóes dos familiares (Zwaigenbaum et al., 2019).

Aragão et al. (2018) abordaram, em seu estudo, os aspectos sensoriais do bebê no contexto hospitalar sem fazer o uso de instrumentos padronizados de avaliação. As autoras apontam que há uma dificuldade em definir uma padronização na identificação sobre como os estímulos sensoriais são captados pelo bebê (Aragão et al., 2018). Os resultados sugerem que as experiências sensoriais projetadas aos bebês são oriundas de experiências de exploração do próprio corpo e inclusive das diferentes formas de cuidado durante a higienização, a alimentação e o vestuário realizadas por um cuidador. Defender a maneira de cuidar sobre a óptica de uma singularidade sugere que cada família tem a sua maneira de cuidar, frente a sua cultura e experiências diferentes (Beltrame et al., 2018; Peruzzolo \& Souza, 2017), necessitando da ampliação do uso de instrumentos padronizados que considerem os aspectos do bebê, o contexto e a maneira de realizar os cuidados pelos pais no dia a dia (Della Barba, 2018).

\subsection{AspeCtos SENSORIAIS E OUTROS INSTRUMENTOS DE AVALIAÇÃO DO DESENVOLVIMENTO INFANTIL}

Dos instrumentos utilizados nos artigos incluídos nesta revisão, dois contemplam a avaliação de aspectos sensoriais a partir de avaliaçóes dos contextos em que o bebê está inserido, a saber: Home Observation for Measurement of the Environment (HOME) e o Affordance in the Home Environment for Motor Development-Infant Scale (AHEMD-IS).

Ambos os estudos identificados apontaram que a estruturação do ambiente em que a criança está inserida, tanto em casa (Avan et al., 2014) como em contextos escolares (Pedrosa et al., 2015), influenciam o desenvolvimento sensorial das crianças, apesar de ambos não se caracterizarem como instrumentos de avaliação do desenvolvimento sensorial do bebê e não fazerem referência ao impacto do ambiente no desenvolvimento sensorial especificamente.

O objetivo do estudo de Pedrosa et al. (2015), em caracterizar o ambiente quanto à estimulação sensorial e relacionar com o instrumento TSFI, apontou que as estimulaçóes 
realizadas no cotidiano e nos contextos de inserção devem seguir sendo exploradas, já que as oportunidades positivas de estimulação nos contextos de inserção do bebê, como a creche, apontaram bons resultados no TSFI, principalmente quanto à organização de estímulos disponibilizados nos ambientes e ao período de tempo de permanência dos bebês nesses espaços.

Viganó et al. (2014) salientam a importância de considerar espaços como as creches para o desenvolvimento a partir das influências dos aportes sensoriais dos bebês. As autoras identificaram, em seu estudo, que os bebês demonstraram importantes alteraçóes no processamento sensorial dos 7 aos 36 meses, necessitando de estimulaçóes adequadas que venham a apoiar o desenvolvimento infantil. Neste estudo, o instrumento Perfil Sensorial foi respondido pelos cuidadores, porém considerando que muitas famílias brasileiras necessitam inserir os bebês em espaços escolares muito precocemente. Acredita-se que os professores são importantes observadores dos sinais e das respostas dadas pelos bebês frente a diferentes estímulos do dia a dia, o que ampliaria a possibilidade de ofertas dirigidas aos bebês conforme as suas necessidades (Bröring et al., 2018). Essa problemática também é apontada no estudo de Pedrosa et al. (2015), visto que as autoras salientam que a identificação individualizada das necessidades do bebê em espaços escolares possibilita organizar os ambientes conforme as capacidades de cada bebê, atentando-se para o planejamento prévio das atividades pedagógicas, lúdicas e de motricidade.

Três estudos utilizaram a Escala Bayley de Desenvolvimento do bebê e da criança pequena - Bayley III (Avan et al., 2014; Cavaggioni, 2017; Chorna, et al. 2014). Em um deles (Avan et al., 2014), os autores utilizaram a escala HOME associada. Mesmo com a Escala Bayley III tendo como foco a avaliação de habilidades relacionadas à linguagem expressiva e receptiva, motricidade grossa e fina e cognição de bebês, o instrumento reúne em uma de suas baterias de teste um questionário com os pais, a qual se propóe a avaliar o comportamento adaptativo do bebê. Nessa etapa do instrumento, são contemplados alguns itens de avaliação do processamento sensorial por meio de atividades cotidianas do bebê (Cavaggioni, 2017), o que se assemelha aos itens abordados pela escala de avaliação Perfil Sensorial 2 (Almohalha, 2018).

A Escala Bayley III é vista como padrão ouro para identificação de sinais de risco ao desenvolvimento (Madaschi et al., 2016), portanto pode ser uma boa ferramenta para uso de profissionais que têm interesse em estudar os padrôes de desenvolvimento sensorial em bebês, tanto nacional como internacionalmente, já que, no ano de 2016, foram publicados os resultados quanto aos dados normativos para aplicação na população brasileira (Madaschi et al., 2016). Ainda que com a validação da Escala Bayley III, observa-se, no Quadro 1, que, no estudo de Cavaggioni (2017), não foi utilizada a versão do instrumento adaptada para a população brasileira, o que torna necessário o aumento de investigaçóes com as versóes dos instrumentos padronizados para essa população, já que isso implica, de forma significativa, os resultados apresentados em pesquisas (Machado et al., 2017; Madaschi et al., 2016; Mélo et al., 2019).

Chorna et al. (2014) utilizaram o instrumento TSFI com o objetivo de avaliar os aspectos sensoriais e comparar aos aspectos cognitivos identificados pela Escala Bayley aos dois anos. Foi possível identificar que os bebês que obtiveram baixos escores no subteste função adaptativa, no TSFI, apresentaram baixos escores nos aspectos motores e de linguagem no Bayley III. Buffone et al. (2016) também relacionaram as Escalas Bayley e o TSFI para avaliar a relação entre o processamento sensorial e o desempenho cognitivo de bebês prematuros entre 
oito e 15 meses de idade, constatando que as dificuldades cognitivas dos bebês não estavam associadas à prematuridade, porém o atraso cognitivo foi significantemente maior entre os lactentes com processamento sensorial em risco e deficiente, quando comparados aos bebês com processamento sensorial normal.

Considerar a Escala Bayley como um dos instrumentos de avaliação de processamento sensorial a partir dos relatos dos pais vai ao encontro também do que Della Barba (2018) propóe sobre a necessidade de que as intervençóes com a população infantil estejam atreladas às ocupaçóes cotidianas das crianças. Isso requer avaliaçóes de aspectos sensoriais que considerem o contexto em que a criança está inserida, assim como identificado em alguns estudos incluídos nesta revisão (Campos et al., 2010; Cavaggioni, 2017).

Observa-se que não foram encontrados estudos com bebês de 0 a 6 meses que utilizaram instrumentos de avaliação do desenvolvimento psíquico que, atualmente, estão validados para população brasileira como os instrumentos IRDI e PREAUT utilizados por Beltrame et al. (2018) para avaliar a relaçáo dos resultados dos instrumentos com aspectos sensoriais em bebês aos 12 meses. No estudo, é possível identificar que os bebês que apresentaram sinais de risco psíquico nos instrumentos IRDI e/ou PREAUT apresentaram piores escores nos instrumentos de avaliação sensorial. Zwaigenbaum et al. (2019) salientaram algumas recomendações e ferramentas claras por meio de questionários com os pais e sinais no bebê baseadas em evidências para ajudar os pediatras e outros profissionais de saúde, bem como cuidadores a monitorar os primeiros sinais de TEA de forma precoce. As autoras apontaram um passo importante para um diagnóstico preciso e precoce que, segundo os autores, deve levar em consideração as possíveis dificuldades sensoriais dos bebês.

\section{CONSIDERAÇÓES FINAIS}

Destaca-se o ineditismo deste estudo, pois a revisão apresentada indica a necessidade de mais pesquisas voltadas à tradução e à validação tanto dos testes importados como dos instrumentos desenvolvidos no Brasil para avaliação de aspectos sensoriais em bebês do nascimento aos seis meses. Compreende-se que a temática amplia as contribuiçôes para o melhor desempenho de bebês e crianças em risco, nos ambientes escolares regulares ou inclusivos, além de possibilitar a qualificação do desenvolvimento infantil, tornando-o mais favorável para o desempenho e a participação em ocupaçóes cotidianas.

Há uma tendência de que o contexto - tanto os cuidadores quanto os ambientes seja considerado para informaçóes mais amplas quanto ao funcionamento do processamento sensorial dos bebês para criação de novos instrumentos e para o aprimoramento dos estudos futuros sobre aspectos sensoriais em bebês. Além disso, conclui-se que escalas que contemplam o desenvolvimento de aspectos motores e cognitivos e, também, consideram aspectos sensoriais são relevantes para uso em estudos futuros.

A validação e a adaptação de instrumentos de avaliação de aspectos sensoriais de bebês qualificam as avaliaçóes do desenvolvimento infantil, já que consideraria dados culturais e históricos da população brasileira. A identificação padronizada pode aumentar as oportunidades de familiares, profissionais da saúde e da educação em reconhecer as necessidades de apoios 
sensoriais necessários para que cada bebê se desenvolva de forma satisfatória e conquiste suas habilidades gradativamente, caracterizando-se como uma medida de prevenção, de responsabilidade de terapeutas ocupacionais, professores e educadores especiais, que possam evitar ou diminuir as dificuldades no processo de aprendizagem futura.

Além disso, amplia-se a identificação de sinais de risco àqueles bebês que, mesmo com a organização do contexto e dos estímulos oferecidos por professores e pais, necessitam de encaminhamentos para tratamento precoce, diminuindo os efeitos iatrogênicos no desenvolvimento em curto e longo prazo, tanto em grupos de crianças típicas como em grupos de bebês que apresentam fatores de risco como prematuridade, risco psíquico e condições neurológicas.

As principais limitaçóes deste estudo englobam fatores relacionados à caracterização da revisão, tais como: a expansão da revisão com a inserção de literatura cinzenta, a ampliação do período de publicação dos artigos incluídos e a inserção de outros descritores para realização dos cruzamentos nas bases de dados.

\section{REFERÊNCIAS}

Almohalha, L. (2018). Tradução, adaptação cultural e validação do Infant Sensory Profile 2 e do Toddler Sensory Profile 2 para crianças brasileiras de 0 a 35 meses. [Tese de Doutorado, Escola de Enfermagem de Ribeirão Preto, Universidade de São Paulo]. Repositório USP. https://repositorio.usp.br/ item/002897961

Aragão, L. R. F., Maia, F. N., \& Mitre, R. M. A. (2018). Os estímulos sensoriais recebidos por crianças com hospitalização prolongada. Cadernos Brasileiros de Terapia Ocupacional, 26(1), 45-51. http:// doi.org/10.4322/2526-8910.ctoAO1057

Avan, B. I., Raza, S. A., \& Kirkwood, B. R. (2014). A community-based study of early childhood sensory stimulation in home environment associated with growth and psychomotor development in Pakistan. International Journal of Public Health, 59(5), 779-788. https://doi.org/10.1007/ s00038-013-0525-7

Beltrame, V. B., Moraes, A. B., \& Souza, A. P. R. (2018). Perfil sensorial e sua relação com risco psíquico, prematuridade e desenvolvimento motor e de linguagem por bebês de 12 meses. Revista de Terapia Ocupacional da Universidade de São Paulo, 29(1), 8-18. http://www.revistas.usp.br/rto/article/ view/138550

Bröring, T., Königs, M., Oostrom, K. J., Lafeber, H. N., Brugman, A., \& Oosterlaan, J. (2018). Sensory processing difficulties in school-age children born very preterm: an exploratory study. Early Human Development, 117, 22-31. https://doi.org/10.1016/j.earlhumdev.2017.12.003

Buffone, F. R. R. C., Eickman, S. H., \& Lima, M. C. (2016). Processamento sensorial e desenvolvimento cognitivo de lactentes nascidos pré-termo e a termo. Cadernos de Terapia Ocupacional, 24(4), 695703. http://doi.org/10.4322/0104-4931.ctoAO0731

Campos, A. C., Coelho, M. C., \& Rocha, N. A. C. F. (2010). Desempenho motor e sensorial de lactentes com e sem síndrome de Down: estudo piloto. Fisioterapia e Pesquisa, 17(3), 203-208. http://doi.org/10.1590/S1809-29502010000300003 
Cavaggioni, A. P. M. (2017). Influência da via de parto no desenvolvimento infantil: comparaçâo por meio da Escala Bayley III. [Dissertação de Mestrado, Escola de Ciências Médicas e da Saúde, Universidade Metodista de São Paulo]. Biblioteca Digital de Teses e Dissertaçóes - TEDE, Metodista. http:// tede.metodista.br/jspui/handle/tede/1692

Chorna, O., Solomon, J. E., Slaughter, J. C., Stark, A. R., \& Maitre, N. L. (2014). Abnormal sensory reactivity in preterm infants during the first year correlates with adverse neurodevelopmental outcomes at 2 years of age. Archives of Disease in Childhood: Fetal \& Neonatal, 99(6), 75-79. https:// doi.or/10.1136/archdischild-2014-306486

DeGangi, G. A., \& Greenspan, S. I. (1989). The Development of Sensory Functions in Infants. Physical o Occupational Therapy In Pediatrics, 8(4), 21-33. https://doi.org/10.1080/J006v08n04_02

Della Barba, P. C. S. (2018). Intervenção precoce no brasil e a prática dos terapeutas ocupacionais. Revista Interinstitucional Brasileira de Terapia Ocupacional, 2(4), 848-861.

Dunn, W., \& Daniels, D. B. (2002). Initial Development of the Infant/Toddler Sensory Profile. Journal of Early Intervention, 25(1), 27-41. https://doi.org/10.1177/105381510202500104

Echevarría-Guanilo, M. E., Gonçalves, N., \& Romanoski, P. J. (2017). Propriedades psicométricas de instrumentos de medidas: bases conceituais e métodos de avaliação - Parte I. Texto \& Contexto Enfermagem, 26(4), 1-11. https://doi.org/10.1590/0104-07072017001600017

Gioia, P. S., \& Guilhardi, C. (2018). Protocolo comportamental de avaliação e intervençáo precoces para bebês de risco autístico. Revista Brasileira de Terapia Comportamental e Cognitiva, 20(3), 118-135. https://doi.org/10.31505/rbtcc.v20i3.1221

Gómez, C. C., Maya, A. M., \& González, A. C. (2019). Los recién nacidos muy prematuros: dificultades en la escuela. Enfermería Global, 18(3), 554-566. http://doi.org/10.6018/eglobal.18.3.347121

Gonçalves, V. M. G., \& Goto, M. M. F. (2010). Avaliação neurológica de lactantes. In M. V. L. MouraRibeiro, \& V. M. G. Gonçalves (Orgs.), Neurologia do desenvolvimento da criança (2a ed., pp. 253269). Revinter.

Guadarrama-Celaya, F., Otero-Ojeda, G. A., Pliego-Rivero, F. B., Porcayo-Mercado, M. R., RicardoGarcell, J., \& Pérez-Ábalo, M. C. (2012). Screening of Neurodevelopmental Delays in Four Communities of Mexico and Cuba. Public Health Nursing, 29(2), 105-115. https:// doi.org/10.1111/j.1525-1446.2011.00982.x

Joaquim, R. H. V. T., Wernet, M., Leite, A. M., Fonseca, L. M. M., \& Mello D. F. (2018). Interaçóes entre mães e bebês prematuros: enfoque nas necessidades essenciais. Cadernos de Terapia Ocupacional da UFSCar, 26(3), 580-589. https://doi.org/10.4322/2526-8910.ctoAO1051

Machado, A. C. C. P., Oliveira, S. R., Magalhães, L. C., Miranda, D. M., \& Bouzada, M. C. F. (2017). Processamento sensorial no período da infância em crianças nascidas pré-termo: revisão sistemática. Revista Paulista de Pediatria, 35(1), 92-101. https://doi.org/10.1590/1984-0462/;2017;35;1;00008

Madaschi, V., Mecca, T. P., Macedo, E. C., \& Paula, C. S. (2016). Bayley-III Scales of Infant and Toddler Development: Transcultural Adaptation and Psychometric Properties. Paidéia, 26(64), 189-197. http://doi.org/10.1590/1982-43272664201606

Mélo, T. R., Araujo, L. B., Novakoski, K. R. M., \& Israel, V. L. (2019). Sistematização de instrumentos de avaliação para os dois primeiros anos de vida de bebês típicos ou em risco conforme o modelo da CIF. Fisioterapia e Pesquisa, 26(4), 380-393. http://doi.org/10.1590/1809-2950/18026126042019 
Morimoto, S. Y. U., Santos, D. D. A., \& Leite V. M. M. (2020). Atuação do terapeuta ocupacional em uma unidade neonatal do Recife. Revista Interinstitucional Brasileira de Terapia Ocupacional, 4(1), 116-122.

Pedrosa, C., Caçola, P., \& Carvalhal, M. I. M. M. (2015). Fatores preditores do perfil sensorial de lactentes dos 4 aos 18 meses de idade. Revista Paulista de Pediatria, 33(2), 160-166. https://doi. org/10.1016/j.rpped.2014.11.016

Peruzzolo, D. L., \& Souza, A. P. R. (2017). Uma hipótese de funcionamento psicomotor como estratégia clínica para o tratamento de bebês em intervenção precoce. Cadernos Brasileiros de Terapia Ocupacional, 25(2), 427-434. http://doi.org/10.4322/0104-4931.ctoEN0864

Pisacco, N. M., Sperafico, Y. L. S., Costa, A. C., \& Dorneles, B. V. (2016). Intervençóes escolares em alunos com transtorno de déficit de atenção/hiperatividade. In N. T. Rotta, L. Ohlweiler, \& R. S. Riesgo (Orgs.), Transtornos da Aprendizagem - Abordagem Neurobiológica e Multidisciplinar (2a ed., pp. 339-356). Artmed.

Reis, J. C., Santos, P. S., Barata, M. F. O., \& Falcão, I.V. (2018). Abordagem da terapia ocupacional a bebês com microcefalia: uma experiência no estágio curricular. Revista Interinstitucional Brasileira de Terapia Ocupacional, 2(1), 212-227.

Silva, N. D. S. H., Lamy Filho, F., Gama, M. E. A., Lamy, Z. C., Pinheiro, A. L., \& Silva, D. N. (2011). Instrumentos de avaliação do desenvolvimento infantil de recém-nascidos prematuros. Revista Brasileira de Crescimento e Desenvolvimento Humano, 21(1), 85-98. https://doi.org/10.7322/jhgd.19998

Souza, A. P. R., Hoogstraten, A. M. R. V., Rechia, I. C., Silva, M. F. A., Nunes, S. F., \& Santos, T. D. (2019). Linguagem, cognição e psiquismo: análise do brincar de dois bebês com histórico de sofrimento psíquico. Estilos da Clínica, 24(1), 84-97. https://doi.org/10.11606/issn.1981-1624. v24i1p84-97

Souza, M. T., Silva, M. D., \& Carvalho, R. (2010). Revisão integrativa: o que é e como fazer? Einstein, 8(1), 102-106. https://doi.org/10.1590/s1679-45082010rw1134

Ursi, E. S., \& Galvão, C. M. (2006). Prevenção de lesóes de pele no perioperatório: revisão integrativa da literatura. Revista Latino-Americana de Enfermagem, 14(1), 124-131. https://doi.org/10.1590/ S0104-11692006000100017

Viganó, A. G., Domingues, L. P., Mendes, M. F., Silva, M. T. B., \& Lima, M. V. A. F. (2014). Perfil sensorial de crianças de 7 a 36 meses frequentadoras de creches municipais. Pediatria Moderna, 50(3), 106-112.

Zwaigenbaum, L., Brian, J. A., \& Ip, A. (2019). Early detection for autism spectrum disorder in young children. Paediatrics \& Child Health, 24(7), 424-432. https://doi.org/10.1093/pch/pxz119-

Recebido em: 22/07/2020

Reformulado em: 01/09/2020

Aprovado em: 17/10/2020 
BELTRAME, V.H.; HERMES, J.B.; JOAQUIM, R.H.V.T.

\section{Errata}

Na página 411

Onde se lê: "BELTRAM"

Leia-se: "BELTRAME"

$\mathrm{Na}$ legenda das páginas 411 a 426

Onde se lê: BELTRAM, V.H.; HERMES, J.B.; JOAQUIM, R.H.V.T.

Leias-se: BELTRAME, V.H.; HERMES, J.B.; JOAQUIM, R.H.V.T. 